\title{
On uniqueness in the inverse obstacle problem via the positive supersolutions of the Helmholtz equation
}

\author{
Masaru IKEHATA* \\ Final, February 10, 2012
}

\begin{abstract}
This paper is concerned with an inverse obstacle scattering problem of an acoustic wave for a single incident plane wave and a wave number. The Colton-Sleeman theorem states the unique recovery of sound-soft obstacles with a smooth boundary from the far-field pattern of the scattered wave for a single incident plane wave at a fixed wave number. The wave number has a bound given by the first Dirichlet eigenvalue of the negative Laplacian in an open ball that contains the obstacles. In this paper, another proof of the Colton-Sleeman theorem that works also for the case when we have a known unbounded set that contains obstacles is given. Unlike original one, the proof given here is not based on the monotonicity of the first Dirichlet eigenvalue of the negative Laplacian. Instead, it relies on a positive supersolution of the Helmholtz equation in a known domain that contains obstacles. Some corollaries which are new and not covered by the Colton-Sleeman Theorem are also given.
\end{abstract}

AMS: 35R30

KEY WORDS: inverse obstacle scattering, sound-soft, sound wave, Helmholtz equation, supersolution, single incident wave, fixed wave number

\section{Introduction}

This paper is concerned with an inverse obstacle scattering problem of acoustic wave for a single incident plane wave and wave number.

Let $D \subset \mathbf{R}^{m}, m=2,3$ be a bounded domain with $C^{2}$ boundary such that $\mathbf{R}^{m} \backslash \bar{D}$ is connected. The total wave field $u$ outside sound-soft obstacle $D$ takes the form $u(x ; d, k)=$ $e^{i k x \cdot d}+w(x)$ with $k>0, d \in S^{m-1}$ and satisfies

$$
\begin{gathered}
\triangle u+k^{2} u=0 \text { in } \mathbf{R}^{m} \backslash \bar{D}, \\
u=0 \text { on } \partial D, \\
\lim _{r \longrightarrow \infty} r^{(m-1) / 2}\left(\frac{\partial w}{\partial r}-i k w\right)=0 .
\end{gathered}
$$

*Department of Mathematics, Graduate School of Engineering, Gunma University, Kiryu 376-8515, JAPAN 
The last condition above is called the Sommerfeld radiation condition [3].

The scattered wave $w=u-e^{i k x \cdot d}$ for fixed $d$ and $k$ has the asymptotic behaviour

$$
w(r \varphi)=\frac{e^{i k r}}{r^{(m-1) / 2}} F_{D}(\varphi, d ; k)+O\left(\frac{1}{r^{(m+1) / 2}}\right), r \longrightarrow \infty
$$

uniformly for $\varphi \in S^{m-1}$, and coefficient $F_{D}(\varphi, d ; k)$ is called the far-field pattern.

We consider the uniqueness issue of the inverse problem: determine $D$ from $F_{D}(\cdot, d ; k)$ for a fixed $d$ and $k$.

This is a well-known open problem, and the complete answer is yet unknown [5]. However, there is a partial result with a constraint on the range of $k$ depending on an a priori information about the location of $D$ and not the shape. In this paper, we denote by $\lambda_{j, m}(U)$ for a bounded connected open set $U \subset \mathbf{R}^{m}$ the $\mathrm{j}$-th Dirichlet eigenvalue of $-\triangle$ in $U$.

\subsection{A review of the Colton-Sleeman theorem and Gintides's im- provement}

In [2] Colton and Sleeman have established the following theorem which we call the Colton-Sleeman theorem.

Theorem 1.1([2]). Assume that there exists an open ball $B$ with radius $R$ such that $D \subset B$. If $k^{2}<\lambda_{1, m}(B)$, then $D$ is uniquely determined by $F_{D}(\cdot, d ; k)$ for a fixed $d$ and $k$.

Note that $\lambda_{1,3}(B)=(\pi / R)^{2}$ and $\lambda_{1,2}(B)=\left(\gamma_{0} / R\right)^{2}$, where $\gamma_{0}$ is the smallest positive zero of the Bessel function $J_{0}(z)$.

The assumption means that for fixed $k$, the radius of $B$ that contains $D$ which is a priori information about the location of $D$ can not be large. It should be pointed out that the optimal case $k^{2}=\lambda_{1, m}(B)$ is excluded. Their proof does not work for this case.

Their assertion is as follows.

Let $D_{1}$ and $D_{2}$ be two obstacles, and $u_{1}$ and $u_{2}$ denote the corresponding total fields. Let $F_{1}$ and $F_{2}$ be the corresponding far-field patterns for fixed $d$ and $k$. If $F_{1}=F_{2}$, then $D_{1}=D_{2}$.

They employ a contradiction argument which is a traditional one in proving several uniqueness theorems in inverse obstacle scattering and goes back to Schiffer's idea.

The proof can be divided into five steps.

Step 1. Assume that the conclusion is not true: $D_{1} \neq D_{2}$.

Step 2. Showing $u_{1}=u_{2}$ in $D^{\infty}$ with the use of the Rellich lemma [3], where $D^{\infty}$ denotes the unbounded connected component of the set $\mathbf{R}^{m} \backslash\left(\bar{D}_{1} \cup \bar{D}_{2}\right)$.

Step 3. Showing the existence of a nonempty connected open set $D_{\star} \subset D_{\infty} \backslash \bar{D}_{1}$ such that $u_{1}=0$ on $\partial D_{\star}$, where $D_{\infty}=\mathbf{R}^{m} \backslash \overline{D^{\infty}}$ and, if necessary, changing of the role of $u_{1}$ and $u_{2}$. See [5] for this point.

Step 4. Showing $\left.u_{1}\right|_{D_{\star}} \in H_{0}^{1}\left(D_{\star}\right)$. This means that there exists a sequence of smooth functions $\varphi_{n} \in C_{0}^{\infty}\left(D_{\star}\right)$ such that $\varphi_{n} \longrightarrow u_{1}$ in $H_{0}^{1}\left(D_{\star}\right)$. This is because of the general fact: if $U$ is an arbitrary bounded connected open set and $\varphi \in H^{1}(U) \cap C^{0}(\bar{U})$ satisfies $\varphi=0$ on $\partial U$, then $\varphi \in H_{0}^{1}(U)$ (cf. Corollary 3.28 of [6]). Note that the boundary of $D_{\star}$ 
can be wild in general and thus one can not use the characterization of $H_{0}^{1}\left(D_{\star}\right)$ by the trace operator onto $\partial D_{\star}$. See also $[3,7]$ for this point.

Step 5. Showing $k^{2} \geq \lambda_{1, m}\left(D_{\star}\right)$. This is because of $u_{1} \neq \equiv 0$ and $\triangle u_{1}+k^{2} u_{1}=0$ in $D_{\star}$.

Step 6. Showing $\lambda_{1, m}\left(D_{\star}\right) \geq \lambda_{1, m}(B)$. This is because of $D_{\star} \subset B$ and the monotonicity of the first Dirichlet eigenvalue with respect to the domain which is an implication of the mini-max principle for eigenvalues(the Rayleigh-Ritz formula).

From the last step we have $k^{2} \geq \lambda_{1, m}(B)$. Contradiction.

Note that in Step 6 one can not say more like $\lambda_{1, m}\left(D_{\star}\right)>\lambda_{1, m}(B)$. This is the reason why the case $k^{2}=\lambda_{1, m}(B)$ is excluded.

Gintides [4] improved the restriction on $k$ in Theorem 1.1 as

$$
k^{2}<\lambda_{2, m}(B) \text {. }
$$

His argument after having Step 4 is based on the following four facts.

- $\overline{u_{1}}$ is also satisfies the same Helmholtz equation.

- $\overline{u_{1}}$ and $u_{1}$ are linearly independent.

- the dimension of the first Dirichlet eigenspace is one. This is the Courant nodal theorem. See, e.g., on page 133 of [6] for the proof for a bounded domain without any regularity assumption on the boundary just like $D_{\star}$.

- the monotonicity of the second Dirichlet eigenvalue with respect to the domain.

From these he concludes $k^{2} \geq \lambda_{2, m}\left(D_{\star}\right) \geq \lambda_{2, m}(B)$. Contradiction.

All the argument stated above are based on the multiplicity of the eigenvalues and their monotonicity with respect to the domain.

Remark 1.1. Note also that, instead of the monotonicity $\lambda_{1, m}\left(D_{\star}\right) \geq \lambda_{1, m}(B)$, Stefanov and Uhlmann in [7] used an implication of the Poincaré inequality, that is,

$$
\omega_{m} \leq\left(\lambda_{1, m}\left(D_{\star}\right)\right)^{m / 2} \operatorname{Vol}\left(D_{\star}\right),
$$

where $\omega_{m}$ denotes the volume of the unit ball in $\mathbf{R}^{m}$. They proved a uniqueness theorem at fixed $k$ and $d$ provided $D$ contains a known obstacle $D_{-}$and is contained in a known obstacle $D_{+}$and $\operatorname{Vol}\left(D_{+} \backslash D_{-}\right)<\omega_{m} k^{-m}$.

\subsection{Statement of the results}

In this paper, we present another method which is based on a real-valued special function $v$ satisfying $\triangle v+k^{2} v \leq 0$ in a domain that contains unknown obstacles.

Our main result is the following theorem.

Theorem 1.2. Let $\Omega$ be an open connected set with $\bar{D} \subset \Omega$. Let $k_{0}>0$. Assume that there exists a real-valued function $v \in C^{2}(\Omega)$ such that $\Delta v+k_{0}^{2} v \leq 0$ in $\Omega$ and $v(x)>0$ for all $x \in \Omega$. If $k \leq k_{0}$, then $D$ is uniquely determined by $F_{D}(\cdot, d ; k)$ for a fixed $d$ and $k$.

Note that $\Omega$ can be unbounded; it is assumed that $\bar{D} \subset \Omega$ not $D \subset \Omega$. The $v$ in Theorem 1.2 should be called a supersolution of the Helmholtz equation in $\Omega$ at wave number $k_{0}$ (cf. [6] for the notion of the supersolution). Thus Theorem 1.2 can be considered as an application of the supersolution in inverse obstacle scattering problems. The following corollary corresponds to Theorem 1.1 including the case when $k^{2}=\lambda_{1, m}(\Omega)$.

Corollary 1.1. Let $\Omega$ be a bounded open connected set with $\bar{D} \subset \Omega$. If $k^{2} \leq \lambda_{1, m}(\Omega)$, then $D$ is uniquely determined by $F_{D}(\cdot, d ; k)$ for a fixed $d$ and $k$. 
Proof. Let $\phi$ be the first Dirichlet eigenfunction for the negative Laplacian in $\Omega$. By the Courant nodal theorem, one may assume that $\phi(x)>0$ for all $x \in \Omega$. Thus, from Theorem 1.2 with $k_{0}^{2}=\lambda_{1, m}(\Omega)$ and $v=\phi$, one obtains the desired uniqueness result.

However, this fact itself is not new since the result is a special case of the result by Gintides [4] as mentioned in Subsection 1.1 under the condition $k^{2}<\lambda_{2, m}(B)$ and $D \subset \Omega \equiv B$.

Example 1. Let $B=\left\{x \in \mathbf{R}^{m}|| x \mid<R\right\}$. For $\Omega=B$ one can choose

$$
\phi(x)= \begin{cases}J_{0}\left(k_{0}|x|\right), & \text { if } m=2, \\ \frac{\sin k_{0}|x|}{|x|}, & \text { if } m=3,\end{cases}
$$

where $k_{0}=\lambda_{1, m}(B)$.

When $\Omega$ is bounded, one can not find a positive supersolution of the Helmholtz equation in $\Omega$ at the wave number $k>\sqrt{\lambda_{1, m}(\Omega)}$. This is because of the following fact.

Proposition 1.1. Let $\Omega$ be a bounded open connected set of $\mathbf{R}^{m}$. There exists a realvalued function $v \in C^{2}(\Omega)$ such that $\triangle v+k^{2} v \leq 0$ in $\Omega$ and $v(x)>0$ for all $x \in \Omega$ if and only if $k^{2} \leq \lambda_{1, m}(\Omega)$.

For the proof see Appendix. Thus Theorem 1.2 does not yield a new result beyond the Colton-Sleeman theorem and Gintides's result when $\Omega$ is bounded. However, when $\Omega$ is unbounded, there is a possibility of having a positive supersolution in $\Omega$. This is an advantage of Theorem 1.2. The following two corollaries are new and not covered by the Colton-Sleeman theorem or Gintides's result.

Corollary 1.2. Let $\Omega^{\prime}$ be a bounded open connected set of $\mathbf{R}^{2}$ with $\bar{D} \subset \mathbf{R} \times \Omega^{\prime}$. If $k^{2} \leq \lambda_{1,2}\left(\Omega^{\prime}\right)$, then $D$ is uniquely determined by $F_{D}(\cdot, d ; k)$ for a fixed $d$ and $k$.

Proof. Let $\phi^{\prime}$ be the first positive Dirichlet eigenfunction for the negative Laplacian in $\Omega^{\prime}$. Define $v\left(x_{1}, x_{2}, x_{3}\right)=\phi^{\prime}\left(x_{2}, x_{3}\right)$ for $x \in \mathbf{R} \times \Omega^{\prime}$. This $v$ satisfies $\Delta v+k_{0}^{2} v=0$ in $\mathbf{R} \times \Omega^{\prime}$ with $k_{0}^{2}=\lambda_{1,2}\left(\Omega^{\prime}\right)$ and $v(x)>0$ for all $x \in \mathbf{R} \times \Omega^{\prime}$.

Example 2. Let $\left.\Omega^{\prime}=\right]-R, R[\times]-h, h[$ with $h, R>0$. Then

$$
\lambda_{1,2}\left(\Omega^{\prime}\right)=\left(\frac{\pi}{2}\right)^{2}\left(\frac{1}{h^{2}}+\frac{1}{R^{2}}\right)
$$

and

$$
\left.\phi^{\prime}\left(x_{2}, x_{3}\right)=\cos \frac{\pi}{2 R} x_{2} \cos \frac{\pi}{2 h} x_{3},\left(x_{2}, x_{3}\right) \in\right]-R, R[\times]-h, h[.
$$

Thus the condition $k^{2} \leq \lambda_{1,2}\left(\Omega^{\prime}\right)$ is equivalent to

$$
k \leq \frac{\pi}{2} \sqrt{\frac{1}{h^{2}}+\frac{1}{R^{2}}} .
$$

A similar idea yields

Corollary 1.3. Let $J$ be a bounded open interval of $\mathbf{R}$ with $\bar{D} \subset \mathbf{R}^{2} \times J$. If $k^{2} \leq \lambda_{1,1}(J)$, then $D$ is uniquely determined by $F_{D}(\cdot, d ; k)$ for a fixed $d$ and $k$. 
Example 3. Let $J=]-h, h\left[\right.$ with $h>0$. Then $\lambda_{1,1}(J)=(\pi / 2 h)^{2}$ and an associated positive Dirichlet eigenfunction for the negative Laplacian in $J$ is given by

$$
\phi\left(x_{3}\right)=\cos \frac{\pi}{2 h} x_{3},\left|x_{3}\right|<h .
$$

The condition $k^{2} \leq \lambda_{1,1}(J)$ is equivalent to

$$
k \leq \frac{\pi}{2 h}
$$

Examples 2 and 3 suggest that the larger is the number of unbounded directions of the domain $\Omega$, the lower is the upper bound $k_{0}$.

\section{Proof of Theorem 1.2}

We start with describing a well known identity.

Proposition 2.1. Let $u$ and $v$ be arbitrary smooth functions on an open set $U$ and satisfy $v(x) \neq 0$ for all $x \in U$. Then we have

$$
\nabla \cdot\left(v^{2} \nabla \varphi\right)=v \triangle u-u \triangle v \text { in } U
$$

where

$$
\varphi=\frac{u}{v}
$$

Using this identity, we have the following lemma.

Lemma 2.1. Let $k_{0}>0$. Assume that there exists a real-valued function $v \in C^{2}(\Omega)$ such that $\triangle v+k_{0}^{2} v \leq 0$ in $\Omega$ and $v(x)>0$ for all $x \in \Omega$. Let $U$ be a bounded open connected set of $\Omega$ with $\bar{U} \subset \Omega$. If $k \leq k_{0}$ and $u \in C^{2}(U) \cap C^{0}(\bar{U})$ satisfies

$$
\begin{gathered}
\triangle u+k^{2} u=0 \text { in } U, \\
u=0 \text { on } \partial U
\end{gathered}
$$

then $u=0$ in $U$.

Proof. Define $\varphi=u / v$ in $U$. It follows from Proposition 2.1 that

$$
\nabla \cdot\left(v^{2} \nabla \varphi\right)+\left(\triangle v+k^{2} v\right) v \varphi=0 \text { in } U
$$

Since $v^{2}$ has a positive uniform lower bound on $U, \varphi=0$ on $\partial U$ and

$$
\left(\triangle v+k^{2} v\right) v=\left(\triangle v+k_{0}^{2} v\right) v-\left(k_{0}^{2}-k^{2}\right) v^{2} \leq 0 \text { in } U
$$

the weak maximum principle $([6])$ yields $\varphi=0$ in $U$ and thus $u=0$ in $U$.

The proof of Theorem 1.2 starts with having Step 3. Applying Lemma 2.1 with $u=u_{1}$ and $U=D_{\star}$, we have $u_{1}=0$ in $D_{\star}$. Then the unique continuation gives $u_{1}=0$ in $\mathbf{R}^{m} \backslash \bar{D}_{1}$ and this contradicts $u_{1} \sim e^{i k x \cdot d}$ as $|x| \longrightarrow \infty$. Therefore it must hold that $D_{1}=D_{2}$. 
Remark 2.1. Note that: if one starts with having Step 4 in Subsection 1.1, then $\varphi=$ $u_{1} /\left.v\right|_{D_{\star}} \in H_{0}^{1}\left(D_{\star}\right)$. Using $(2.1)$ and a sequence in $C_{0}^{\infty}\left(D_{\star}\right)$ that converges to $\varphi$ in $H_{0}^{1}\left(D_{\star}\right)$, we have

$$
\int_{D_{\star}} v^{2}|\nabla \varphi|^{2} d x-\int_{D_{\star}}\left(\triangle v+k^{2}\right) v|\varphi|^{2} d x=0 .
$$

This also yields the same conclusion as above. This avoids the use of the weak maximum principle, however, needs a knowledge that $\left.u_{1}\right|_{D_{\star}} \in H_{0}^{1}\left(D_{\star}\right)$.

Remark 2.2. The argument done in the proof of Lemma 2.1 together with the use of Proposition 2.1 are well-known typical one in studying the maximum principle for general elliptic partial differential equations (e.g., [6] and introduction of [1]). Here we presented it just for the use of (2.1) in Remark 2.1, i.e., its use in inverse obstacle scattering problems.

\section{Conclusion}

The previous known proof of the Colton-Sleeman and Gintides's improvement are based on some facts on the Dirichlet eigenvalues of the negative Laplacian in a domain and their monotone dependence on domains.

Our proof is extremely simple and uses a positive supersolution $v$ of the Helmholtz equation in a domain $\Omega$ that contains the closure of unknown obstacle. Domain $\Omega$ in three dimensions can be unbounded for a single direction as shown in Corollary 1.2 and two directions as in Corollary 1.3 if the wave number has a bound depending on the size of the "bounded part" of $\Omega$.

\section{Acknowledgement}

This research was partially supported by Grant-in-Aid for Scientific Research (C)(No. 21540162) of Japan Society for the Promotion of Science. The author would like to thank a referee who made constructive suggestions including the change of the title of the paper.

\section{Appendix. Proof of Proposition 1.1}

First assume the existence of $v$ and that the conclusion is not true. Thus one has $k^{2}>$ $\lambda_{1, m}(\Omega)$. Let $\Omega_{1}, \Omega_{2}, \cdots$ be an exhaustion of $\Omega$ from below in the sense that each $\Omega_{j}$ is open connected and $\bar{\Omega}_{j-1} \subset \Omega_{j} \uparrow \Omega$. Then we have $\lambda_{1, m}\left(\Omega_{j}\right) \geq \lambda_{1, m}(\Omega)$ and $\lambda_{1, m}\left(\Omega_{j}\right) \longrightarrow$ $\lambda_{1, m}(\Omega)$. The latter is also a well known consequence of the Rayleigh-Ritz formula. Thus for a large $j$ we have $k^{2}>\lambda_{1, m}\left(\Omega_{j}\right)$. Let $\phi_{j}$ denote the first Dirichlet eigenfunction for the negative Laplacian in $\Omega_{j}$. Define $\varphi=\phi_{j} / v$ in $\Omega_{j}$. Since $\overline{\Omega_{j}} \subset \Omega, \varphi$ belongs to $C^{2}\left(\Omega_{j}\right) \cap C^{0}\left(\bar{\Omega}_{j}\right)$. It follows from Proposition 2.1 that

$$
\nabla \cdot\left(v^{2} \nabla \varphi\right)+\left(\triangle v+k_{0}^{2} v\right) v \varphi=0 \text { in } \Omega_{j}
$$

where $k_{0}^{2}=\lambda_{1, m}\left(\Omega_{j}\right)$. Since $\varphi=0$ on $\partial \Omega_{j}$ and $\left(\triangle v+k_{0}^{2} v\right) v=\left(\triangle v+k^{2} v\right) v+\left(k_{0}^{2}-k^{2}\right) v^{2} \leq$ 0 in $\Omega_{j}$, the maximum principle yields $\varphi=0$ in $\Omega_{j}$; however, this is impossible by the Courant nodal theorem. Therefore it must hold that $k^{2} \leq \lambda_{1, m}(\Omega)$. Conversely if $k^{2} \leq \lambda_{1, m}(\Omega)$, then choose the first positive Dirichlet eigenfunction $\phi$ for the negative 
Laplacian in $\Omega$ and set $v=\phi$. Then $\triangle v+k^{2} v=\Delta \phi+k_{1}^{2} \phi+\left(k^{2}-k_{1}^{2}\right) \phi \leq 0$ in $\Omega$, where $k_{1}^{2}=\lambda_{1, m}(\Omega)$.

\section{References}

[1] Berestycki, H., Nirenberg, L. and Varadhan, S. R. S., The principle eigenvalue and maximum principle for second-order elliptic operators in general domains, Comm. Pure Appl. Math., 47(1994), 47-92.

[2] Colton, D. and Sleeman, B. D., Uniqueness theorems for the inverse problem of acoustic scattering, IMA J. Appl. Math., 31(1983), 253-259.

[3] Colton, D. and Kress, R., Inverse acoustic and electromagnetic scattering theory, Springer, second edition, 1998.

[4] Gintides, D., Local uniqueness for the inverse scattering problem in acoustics via the Faber-Krahn inequality, Inverse Problems, 21(2005), 1195-1205.

[5] Isakov, V., Inverse problems for partial differential equations, Springer, second edition, New York, 2006.

[6] Murata, M. and Kurata, K., Elliptic and parabolic type partial differential equations, in japanese, Iwanami Shoten, 2006, Tokyo.

[7] Stefanov, P. and Uhlmann, G., Local uniqueness for the fixed energy fixed angle inverse problem in obstacle scattering, Proc. AMS., 132(2004), 1352-1354.

e-mail address

ikehata@math.sci.gunma-u.ac.jp 